\title{
Improved Transient Performance of Nonlinear Adaptive Backstepping using Estimator Resetting based on Multiple Models
}

\author{
Jens Kalkkuh1*, Tor A Johansen ${ }^{* * * * * 1}$ and Jens Lüdemann* \\ *DaimlerChrysler Research and Technology, Alt-Moabit 96A, D-10559 Berlin, Germany. \\ ** Department of Engineering Cybernetics,Norwegian University of Science and \\ Technology, N-7491 Trondheim, Norway.
}

*** SINTEF Electronics and Cybernetics, N-7465 Trondheim, Norway.

June 19,2001

\begin{abstract}
It is proved that the transient performance of nonlinear adaptive backstepping can be improved by resetting the parameter estimator, without loss of stability. The estimator resetting algorithm is based on multiple model adaptive control, where a number of models with fixed parameter vectors are monitored online in order to detect parameter vectors that gives a negative jump in the control Lyapunov function when replacing the estimate provided by the standard adaptation law. Application to wheel slip control is studied.
\end{abstract}

\section{Introduction}

The use of multiple models to switch or reset parameter estimators has been proposed in order to speed up the convergence-rate in the context of certainty equivalence adaptive control of linear systems $[8,9,6,7,5$, 10]. Here we apply multiple models, each with an a priori given fixed parameter vector, in order to reset the estimate of nonlinear backstepping adaptive control law. Such modifications are of particular importance

${ }^{1}$ Corresponding author: Tor.Arne.Johansen@itk.ntnu.no 
in many applications where the environment or system parameters changes rapidly or instantaneously.

This paper contains three main contributions. First, the multiple model adaptive control idea is extended to a class of nonlinear systems, where a conventional adaptive control system is first designed using control Lyapunov functions and backstepping [4]. The resetting algorithms required by the backstepping design are somewhat different from the ones suggested in $[8,9,6,7]$ for linear systems, since backstepping does not rely on the certainty equivalence principle. Second, it is shown that adding a resetting algorithm increases the convergence-rate without loss of stability. Notice that in the above references only proofs of stability are given, and transient performance is not investigated theoretically in these references although it is stated as the main motivation of the methods. We also discuss uncertainty (such as modelling error and noise) in the resetting algorithm since this constitutes a major limitation on the achievable performance. Thirh, we describe an idealized application to wheel slip control (which is the core of automotive ABS brakes) where the multi-model reset algorithm is applied to improve the transient performance in the case of instantaneous changes in the road/tire friction coefficient. This is important when braking on a heterogeneous road surface, for example one that is partly covered with ice or water.

\section{Nonlinear adaptive backstepping}

Adaptive backstepping design for an uncertain parametric strict-feedback system is reviewed, [4]:

$$
\begin{aligned}
\dot{x}_{1} & =x_{2}+\varphi_{1}\left(x_{1}\right)^{T} \theta \\
\dot{x}_{2} & =x_{3}+\varphi_{2}\left(x_{1}, x_{2}\right)^{T} \theta \\
& \vdots \\
\dot{x}_{n-1} & =x_{n}+\varphi_{n-1}\left(x_{1}, x_{2}, \ldots, x_{n-1}\right)^{T} \theta \\
\dot{x}_{n} & =\beta(x) u+\varphi_{n}(x)^{T} \theta
\end{aligned}
$$

where $\theta$ is a vector of unknown constant parameters, and $\beta, \varphi_{1}, \varphi_{2}, \ldots, \varphi_{n-1}$ are smooth functions. The backstepping adaptive controller for this system is given by

$$
\begin{aligned}
& u=\frac{\alpha_{n}(x, \hat{\theta})}{\beta(x)} \\
& \dot{\hat{\theta}}=\Gamma \tau_{n}(x, \hat{\theta})
\end{aligned}
$$


where $\Gamma=\Gamma^{T}>0$ is arbitrary and the control law $\alpha_{n}$ and the tuning function $\tau_{n}$ are given by the following recursive equations:

$$
\begin{aligned}
z_{i} & =x_{i}-\alpha_{i-1} \\
\alpha_{i}\left(x_{1}, \ldots, x_{i}, \hat{\theta}\right) & =-z_{i-1}-c_{i} z_{i}-w_{i}^{T} \hat{\theta}+\sum_{k=1}^{i-1} \frac{\partial \alpha_{i-1}}{\partial x_{k}} x_{k+1}+\frac{\partial \alpha_{i-1}}{\partial \hat{\theta}} \Gamma \tau_{i}+\sum_{k=2}^{i-1} \frac{\partial \alpha_{k-1}}{\partial \hat{\theta}} \Gamma w_{i} z_{k} \\
\tau_{i}\left(x_{1}, \ldots, x_{i}, \hat{\theta}\right) & =\tau_{i-1}+w_{i} z_{i} \\
w_{i}\left(x_{1}, \ldots, x_{i}, \hat{\theta}\right) & =\varphi_{i}-\sum_{k=1}^{i-1} \frac{\partial \alpha_{i-1}}{\partial x_{k}} \varphi_{k}
\end{aligned}
$$

and $\alpha_{0}=0, \tau_{0}=0, c_{i}>0$. The vector containing the vectors $z=\left(z_{1}, z_{2}, \ldots, z_{n}\right)^{T}$ and $\hat{\theta}$ can be interpreted as a transformation of the state composed of $x$ and $\hat{\theta}$. In other words, $z$ depends on $x$ and $\hat{\theta}$. The above adaptive control design is based on the control Lyapunov function

$$
V(x, \hat{\theta})=\frac{1}{2} z^{T} z+\frac{1}{2}(\hat{\theta}-\theta)^{T} \Gamma^{-1}(\hat{\theta}-\theta)
$$

Along trajectories of the closed loop system the control Lyapunov function satisfies the nominal stability condition $\dot{V}=-z^{T} C z$ where $C=\operatorname{diag}\left(c_{1}, \ldots, c_{n}\right)>0$. The present paper extends this design by allowing the parameter estimate to be reset instantaneously from $\hat{\theta}(t)$ to $\hat{\theta}\left(t^{+}\right)$at any time instant $t$. Notice that $t^{+}$denotes an infinitely small time increment of $t$. At these instants in time the parameter update law (3) does not apply, and the control Lyapunov function may be discontinuous as a function of time. Below, we formulate reset conditions that ensures that the control Lyapunov function will make a negative jump at these time instants. Thus, permitting parameter estimator reset will increase the (local) convergence rate of the parameter estimator. In fact, this is the main motivation behind such a reset mechanism.

\section{Estimator reset algorithms}

Suppose the parameter estimate is reset at time $t$. Then the jump in the Lyapunov function is given by

$$
\begin{aligned}
\Delta V(t)= & V\left(x(t), \hat{\theta}\left(t^{+}\right)\right)-V(x(t), \hat{\theta}(t)) \\
= & \frac{1}{2}\left(z^{T}\left(t^{+}\right) z\left(t^{+}\right)-z^{T}(t) z(t)\right)-\frac{1}{2}\left(\hat{\theta}\left(t^{+}\right)-\hat{\theta}(t)\right)^{T} \Gamma^{-1}\left(\hat{\theta}\left(t^{+}\right)-\hat{\theta}(t)\right) \\
& \quad+\left(\hat{\theta}\left(t^{+}\right)-\hat{\theta}(t)\right)^{T} \Gamma^{-1}\left(\hat{\theta}\left(t^{+}\right)-\theta\right)
\end{aligned}
$$

A stability-preserving and performance-improving reset condition is now $\Delta V(t)<0$, see [1]. However, in order to be able to evaluate (10) we need to deal with the fact that $\theta$ in the third term is unknown. Notice that since $z$ depends on $x$ and $\hat{\theta}$, but not on $\theta$, the two first terms of $(10)$ can be evaluated directly. As in conventional adaptive control [3, 4], we introduce some linearly parameterized model that relates 
the parameter error to some prediction error. We assume a finite number of fixed parameter hypotheses $\hat{\theta}_{1}, \ldots, \hat{\theta}_{k}$ that we compare at each time instant to see which one gives the largest guaranteed decrease in $\Delta V(t)$. More precisely, consider a linear parametric model of the form

$$
y(t)=\zeta^{T}(t) \theta
$$

where $y(t) \in R^{m}$ is an output vector and $\zeta(t) \in R^{p \times m}$ is a regressor matrix. This model can be constructed in many ways, see section 6 of [4] for a general result. For simplicity, we consider a first order system $\dot{x}=\varphi^{T}(x) \theta+u$. One may define $y(t)=s H(s) x(t)-H(s) u(t)$ and $\zeta(t)=H(s) \varphi(x(t))$, where $H(s)$ is typically a lowpass or bandpass filter. The main purpose of this filter is replace differentiation operations by apropriate high-pass filters, in addition to reducing the effect of high-frequency noise and low-frequency disturbances in the estimation model. The finite number of fixed parameter hypotheses $\hat{\theta}_{i}$ are now assessed using the predictors

$$
\hat{y}_{i}(t)=\zeta^{T}(t) \hat{\theta}_{i}
$$

for all $i=1,2, \ldots, k$. Notice that because the parameter hypotheses $\hat{\theta}_{i}$ are fixed and $\theta$ is assumed to be time-invariant, the introduction of a filter $H(s)$ as outlined above does not lead to so-called swapping terms $[3]$ in (11) and (12). Prediction errors are defined as

$$
e_{i}(t)=y(t)-\hat{y}_{i}(t)=\zeta^{T}(t)\left(\theta-\hat{\theta}_{i}\right)
$$

The signal $e_{i}(t)$ is available, and contains information about $\theta$. In order to achieve invertability, this equation is premultiplied by the matrix $\zeta(t)$ and integrated on the time interval from $t-T$ to $t$, where $T>0$ is a finite time window:

$$
\int_{t-T}^{t} \zeta(\tau) e_{i}(\tau) d \tau=\int_{t-T}^{t} \zeta(\tau) \zeta^{T}(\tau) d \tau\left(\theta-\hat{\theta}_{i}\right)
$$

Notice that all signals are defined to be zero for $t<0$. Eq. (14) can we written

$$
d_{i}(t)=R(t)\left(\theta-\hat{\theta}_{i}\right)
$$

with the following definitions:

$$
\begin{aligned}
& d_{i}(t)=\int_{t-T}^{t} \zeta(\tau) e_{i}(\tau) d \tau \\
& R(t)=\int_{t-T}^{t} \zeta(\tau) \zeta^{T}(\tau) d \tau
\end{aligned}
$$

Assuming persistence of excitation, i.e. $R(t)>0$, the following expression follows from (15) by inversion:

$$
\theta-\hat{\theta}_{i}=R^{-1}(t) d_{i}(t)
$$


The time window $T$ should be selected to address the tradeoff between noise sensitivity, persistence of excitation and estimator transients. In general, a large $T$ will reduce the effect of uncertainty, but at the cost of a delay in the reset algorithm. Substituting (18) into (10) leads to

$$
\Delta V_{i}(t)=\frac{1}{2}\left(z^{T}\left(t^{+}\right) z\left(t^{+}\right)-z^{T}(t) z(t)\right)-\frac{1}{2}\left(\hat{\theta}_{i}-\hat{\theta}(t)\right)^{T} \Gamma^{-1}\left(\hat{\theta}_{i}-\hat{\theta}(t)+2 R^{-1}(t) d_{i}(t)\right)
$$

Thus, we have proven the following result:

Theorem 1 Suppose the parameter estimate is reset at time $t$ to $\hat{\theta}\left(t^{+}\right)=\hat{\theta}_{i}$ only if $\Delta V_{i}(t) \leq 0$ and $R(t)>0$. Then the adaptive control system with estimator resetting satisfies

$$
\dot{V}(t) \leq-z^{T}(t) C z(t)
$$

where $\dot{V}(t)$ is interpreted in the usual distributional sense when $V(t)$ is discontinuous.

Remark. This result means that resetting may only cause negative jumps in $V(t)$. If there exist several $\hat{\theta}_{i}$ such that $\Delta V_{i}(t)<0$, it makes sense to select the one which minimizes $\Delta V_{i}(t)$ (with respect to $i$ ) since this will yield the largest negative jump in the Lyapunov function. If additional (possibly heuristic) reset conditions are introduced, they can not lead to instability since they will only lead to less frequent switching.

Remark. This reset criterion contains some monitoring of persistence of excitation. If the matrix $R(t)$ becomes close to singular (i.e. the system is poorly excitated) the prediction error must be very small to justify a reset. In the singular case, no reset is allowed. The requirement of persistence of excitation is natural since an instantaneous reset obviously requires that the data contains strong evidence about the parameters. However, this is not an unreasonable requirement since the purpose of the resetting is only to improve transient performance, and the persistence of excitation condition will typically hold during transients. On the other hand, it will typically not hold during close to steady-state conditions, but then there is typically no need for resetting.

Remark. For a first order system, if only one parameter being estimated, it is easily seen that the reset condition $\Delta V_{i}(t)<0$ is equivalent to

$$
\theta-|\hat{\theta}(t)-\theta|<\hat{\theta}_{i}<\theta+|\hat{\theta}(t)-\theta|
$$

This makes intuitively sense, since it requires that $\hat{\theta}_{i}$ is closer to $\theta$ than $\hat{\theta}(t)$. Hence, minimization of $\Delta V_{i}(t)$ is equivalent to minimizing $\left|\theta-\hat{\theta}_{i}\right|$. In this special case, it can also be shown that minimizing the cost 
function introduced in $[8,9]$

$$
J_{i}(t)=\alpha e_{i}^{T}(t) e_{i}(t)+\int_{0}^{t} \exp (-\lambda(t-\tau)) e_{i}^{T}(\tau) e_{i}(\tau) d \tau
$$

is equivalent to minimizing $\Delta V_{i}(t)$ for any $\alpha \geq 0$ and $\lambda>0$. However, in the general case with a higher order system the backstepping procedure introduces additional terms that are not proportional to some parameter error. Hence, the use of the criterion (22) is not always sufficient to guarantee a non-increasing Lyapunov function with the backstepping design. This is not unexpected, since the adaptive control design using backstepping does not rely on the principle of certainty equivalence which is underlying (22).

Remark. Notice that in case filters are used to produce $y(t)$ and $\zeta(t)$ in $(11)$ and $(12)$, there are some exponentially decaying terms in the equations due to the filters' initial conditions that have been neglected in the analysis so far. These terms will, like the introduction of the integral in (14), prevent the reset algorithm from responding instantaneously. Thus, the filters should be tuned to address the tradeoff between response time and sensitivity to uncertainty. This aspect will be discussed in detail in section 4 when considering a specific example.

Remark. Notice that like in (22) one might introduce exponential weighting and an additional weight on the current time instant in (14). This would give more tuning parameters for performance and robustness, but will have no consequences for the results except some simple modifications of the equations.

Remark. Uncertainty may obviously lead to undesirable resetting that may increase the Lyapunov function. For this reason one will in practice introduce some hysteresis $\rho>0$ and require

$$
\Delta V_{i}(t) \leq-\rho
$$

as a reset condition. This will give some margin for uncertainty and give robustness.

\section{Example: Wheel slip control}

A simple model of wheel slip dynamics can be expressed is terms of the following first order model, derived from an analytic expression of the friction curve [2]:

$$
\dot{x}=\varphi(x) \theta+u+\nu
$$

The state $x$ is the control error of the slip, which is the relative speed of the wheel and the vehicle. Control of $x$ to zero implies that the wheel does not lock during braking, and at the same time high braking efficiency is maintained. This is the main objective of an ABS. The control input $u$ is the clamping force, and $\nu$ is 
due to noise and uncertainty. The nonlinear function $\varphi$ is shown in Figure 1, and $\theta$ is the road/tyre friction coefficient. This parameter is highly uncertain and can change instantaneously (as a piecewise constant function of time).

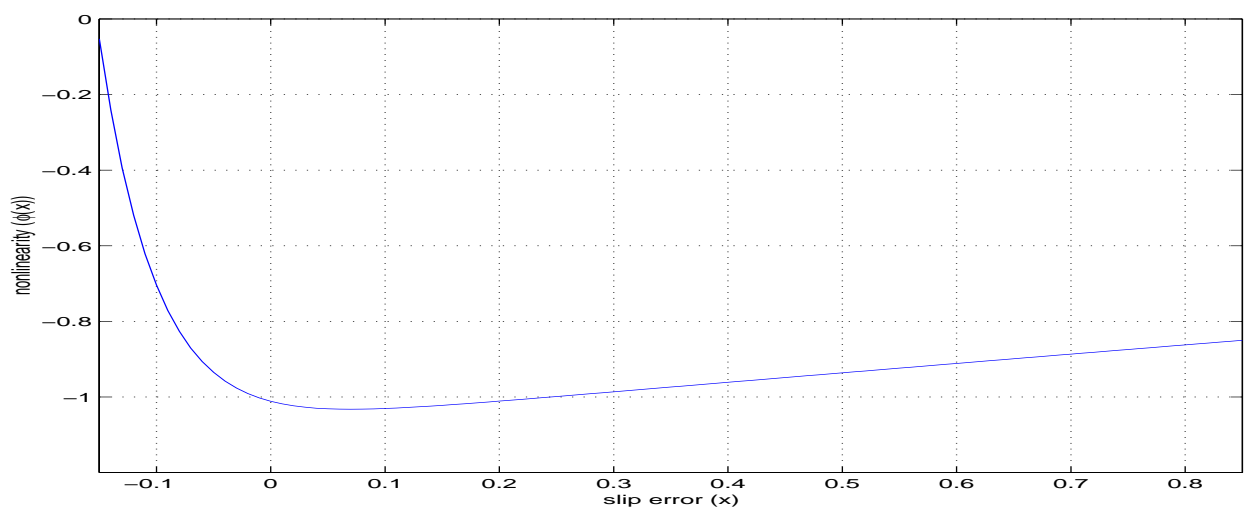

Figure 1: Slip dynamics nonlinearity

In this case the backstepping design leads to the adaptive controller

$$
\begin{aligned}
& u=-c_{1} x-\varphi(x) \hat{\theta} \\
& \dot{\hat{\theta}}=\Gamma \varphi(x) x
\end{aligned}
$$

For the multi-model predictors we apply the same model, with $y(t)=H(s)(s x(t)-u(t))$ and $\zeta(t)=$ $H(s) \varphi(x(t))$. The lowpass filter $H(s)=1 /(1+\tau s)$ has time constant $\tau=0.085$, and the other parameters are $T=0.085, c_{1}=5$ and $\Gamma=10$. There are five parameter hypotheses $\hat{\theta}_{i} \in\{0.9,0.7,0.5,0.3,0.1\}$, and in the simulation there is uniformly distributed white noise $\nu(t) \in[-0.015,0.015]$. Furthermore, we have introduced unmodelled actuator dynamics as a first order system with unit gain and time constant 0.040 in the simulator. Using trial and error, the hysteresis threshold in $(23)$ is chosen as $\rho=0.0015$.

Consider a simulation scenario where the tyre/road friction coefficient $\theta$ changes instantaneously at times $t=2.5$ and $t=5$. Simulation results, with and without estimator resetting, are shown in Figure 2 . It was verified that the persistence of excitation condition $R(t)>0$ was satisfied with a large margin at all time. It can be observed both from the slip error and the values of the Lyapunov function that the performance is indeed improved by the resets.

Note that this scenario differs slightly from the above theoretical setting where the parameter $\theta$ is assumed to be time-invariant. However, these are equivalent if one takes into account the transient generated by the step in $\theta$, as done in the additional analysis below. From Figure 2 it can be observed that the filters and thresholds are tuned such that no erroneous resets are made. Certainly, the filters add transients to the performance signals $\Delta V_{i}(t)$, but these transients have been neglected in the reset algorithm. The rationale 

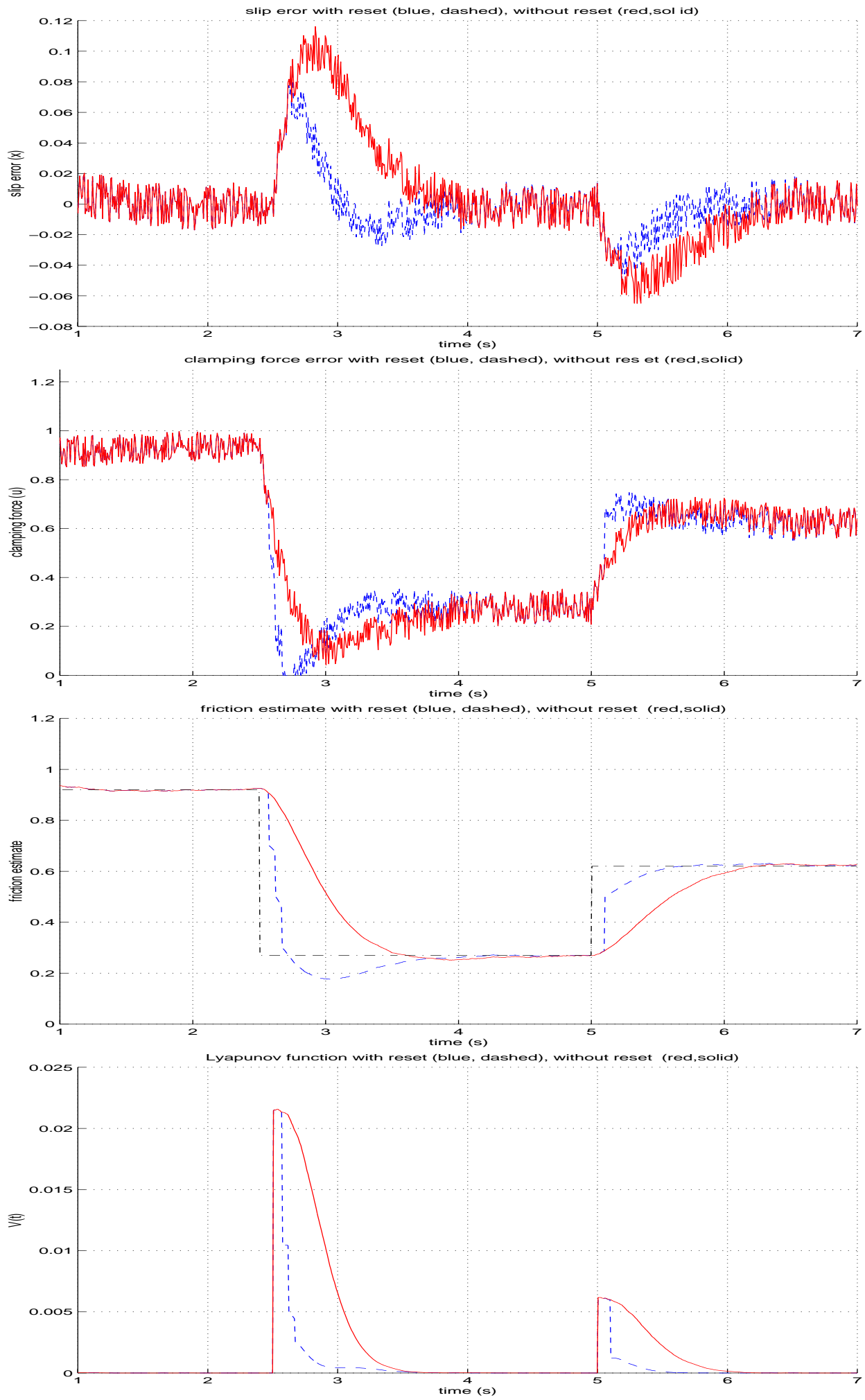

Figure 2: Simulation results. 
for this is shown below.

Consider the instantaneous jump from $\theta=0.92$ to $\theta=0.27$ at time $t=2.5$, i.e.

$$
\theta(t)=0.92-0.65 \mu(t-2.5)
$$

where $\mu(t)$ is the unit step function. This step generates a transient, such that

$$
y(t) \approx 0.92 \zeta(t)-0.65 H(s)(\varphi(x(t)) \mu(t-2.5))
$$

where the uncertainty $\nu(t)$ is neglected. From Figure 1 it is reasonable to assume (for the purpose of simplicity in the analysis) that $\varphi(x(t)) \approx-1$ and consequently $\zeta(t) \approx-1$. Hence,

$$
y(t) \approx-0.92+0.65\left(1-\exp \left(\frac{t-2.5}{0.085}\right)\right)
$$

and

$$
e_{i}(t) \approx\left(-0.27+\hat{\theta}_{i}\right)-0.65 \exp \left(\frac{t-2.5}{0.085}\right)
$$

For $t<2.5$ it was clear that $\hat{\theta}_{1}=0.9$ gave smallest prediction error. For $t>2.5$ it is clear that $\hat{\theta}_{4}=0.3$ is the most accurate estimate. However, compare $e_{4}(t), e_{3}(t)$ and $e_{2}(t)$ (corresponding to $\hat{\theta}_{4}=0.3, \hat{\theta}_{3}=0.5$ and $\hat{\theta}_{2}=0.7$, respectively):

$$
\begin{aligned}
& e_{4}(t) \approx 0.03-0.65 \exp \left(\frac{t-2.5}{0.085}\right) \\
& e_{3}(t) \approx 0.23-0.65 \exp \left(\frac{t-2.5}{0.085}\right) \\
& e_{2}(t) \approx 0.43-0.65 \exp \left(\frac{t-2.5}{0.085}\right)
\end{aligned}
$$

Initially, for $2.50>t>2.56$ it is clear that $\left|e_{2}(t)\right|<\left|e_{3}(t)\right|<\left|e_{4}(t)\right|$ since the first positive term of $e_{2}(t)$ (due to the parameter estimate error) counteracts the negative value of the second term (due to the filter transient), see Figure 3 which illustrates the approximate terms $e_{2}, e_{3}$ and $e_{4}$. Consequently, as seen in Figure 4, the value of $\Delta V_{2}(t)-\rho$ goes negative first, and then $\Delta V_{3}(t)-\rho$ goes negative until finally $\Delta V_{4}(t)-\rho$ goes negative. This can be observed from the plot of $\hat{\theta}(t)$ where it is seen that after $t=2.5$ there is a sequence of three resets to $\hat{\theta}_{2}=0.7, \hat{\theta}_{3}=0.5$ and $\hat{\theta}_{4}=0.3$, all in the right direction. Without filtering and noise, one would instead have a single reset directly to the best value, namely of $\hat{\theta}_{4}=0.3$. To summarize, the filters and their associated transients have the effect that resets are made in several smaller steps rather than in a single big step. One can argue that this is reasonable from a robustness point of view, and thus the filter transient does not necessarily need to be explicitly taken into consideration in the reset algorithm. Thus, although the filter transients will reduce the transient performance, they may tend to improve the robustness of the algorithm. 


\section{Conclusions}

It is shown how to add a stable parameter estimator reset algorithm to a nonlinear adaptive backstepping design. Particular attention is paid to transient performance, which is the main motivation of the additional reset mechanism. The simultion example shows the feasibility and benefits of the approach. The transient performance of a somewhat idealized wheel slip control system has been shown to be significantly improved by adding the reset algorithm.

\section{References}

[1] M. S. Branicky. Multiple Lyapunov functions and other analysis tools for switched and hybrid systems. IEEE Trans. Automatic Control, 43:475-482, 1998.

[2] M. Burckhardt. Fahrwerktechnik: Radschlupfregelsysteme. Vogel-Verlag, Germany, 1993.

[3] P. A. Ioannou and J. Sun. Robust Adaptive Control. Prentice-Hall Inc., 1996.

[4] M. Krstic, I. Kanellakopoulos, and P. Kokotovic. Nonlinear Adaptive Control Design. Wiley and Sons, 1995.

[5] R. H. Middleton, G. C. Goodwin, D. J. Hill, and D. Q. Mayne. Design issues in adaptive control. IEEE Trans. Automatic Control, 33:50-58, 1988.

[6] A. S. Morse. Supervisory control of families of linear set-point controllers - part I: Exact matching. IEEE Trans. Automatic Control, 41:1413-1431, 1996.

[7] A. S. Morse. Supervisory control of families of linear set-point controllers - part II: Robustness. IEEE Trans. Automatic Control, 42:1500-1515, 1997.

[8] K. S. Narendra and J. Balakrishnan. Improving transient response of adaptive control systems using multiple models and switching. IEEE Trans. Automatic Control, 39:1861-1866, 1994.

[9] K. S. Narendra and J. Balakrishnan. Adaptive control using multiple models. IEEE Trans. Automatic Control, 42:141-157, 1997.

[10] P. V. Zhivoglyadov, R. H. Middleton, and M. Fu. Localization based switching adaptive control for time-varying discrete-time systems. IEEE Trans. Automatic Control, 45:752-756, 2000. 


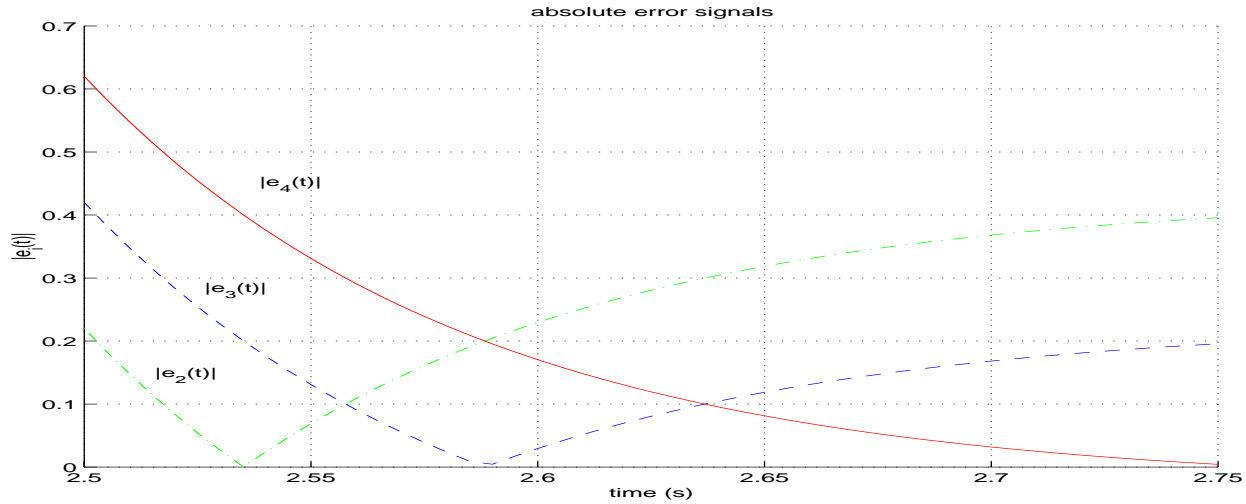

Figure 3: Absolute value of prediction errors after parameter step change. 

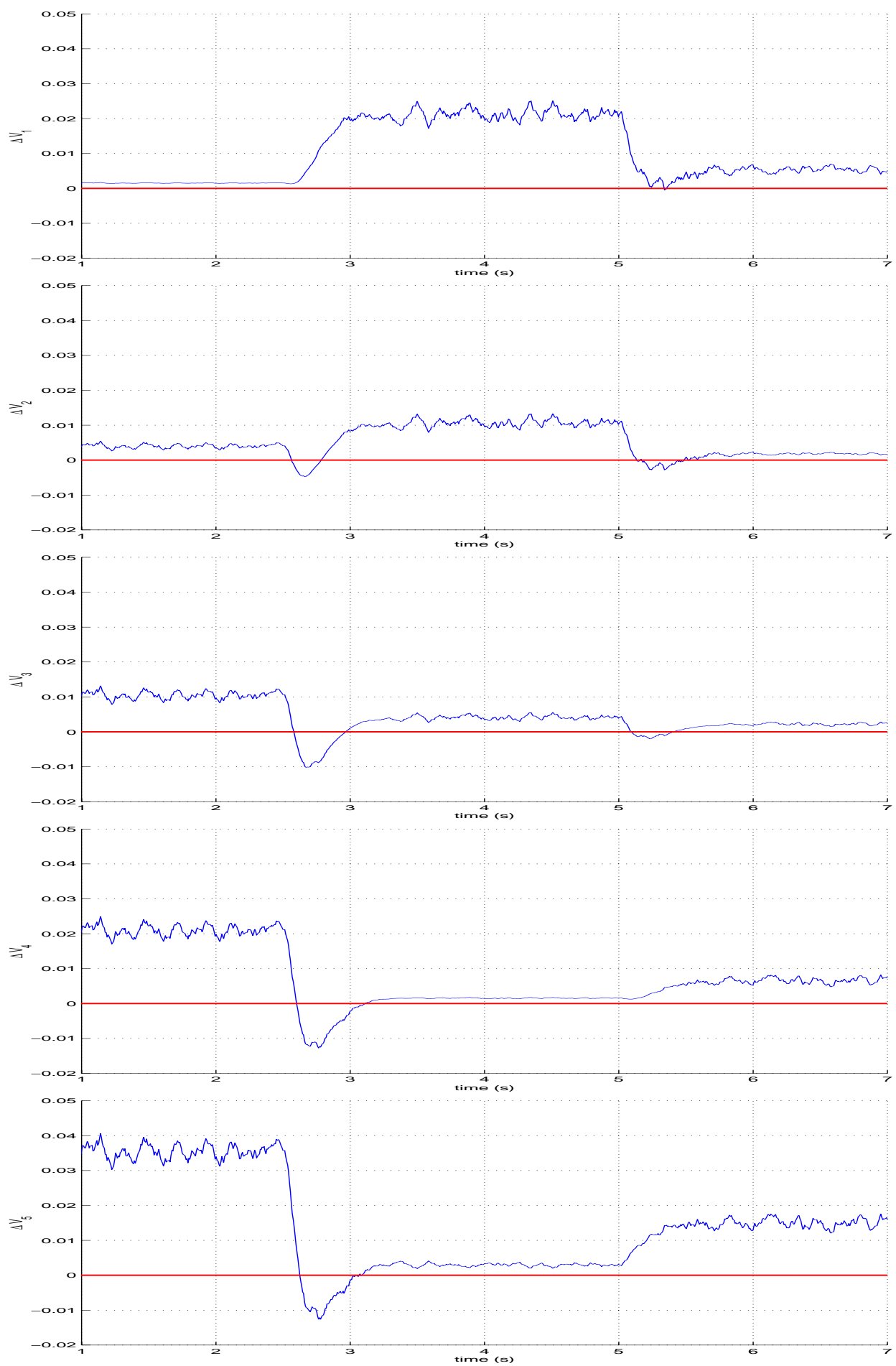

Figure 4: Performance signals for the five models. 